Monatsschr Kinderheilkd 2009 · 157:346-349 DOI 10.1007/s00112-008-1864-y

Online publiziert: 1. April 2009

Redaktion

D. Reinhardt, München

S. Wirth, Wuppertal

R. Haupt ${ }^{1}$ J.J. Spinetta ${ }^{2}$ I. Ban ${ }^{3}$ - R.D. Barr ${ }^{4}$ J.D. Beck . J. Byrne $^{6}$. G. Calaminus ${ }^{7}$. E. Coenen ${ }^{3} \cdot$ M. Chesler ${ }^{3} \cdot$ G.J. D'Angio $^{8} \cdot$ C. Eiser ${ }^{9} \cdot$ A. Feldges ${ }^{10} \cdot$ F. Gibson $^{11}$.

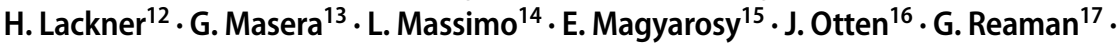
M.G. Valsecchi ${ }^{18}$ - A.J.P. Veerman ${ }^{19}$ - A. Penn ${ }^{20}$ - A. Thorvildsen ${ }^{21}$. C. von den Bos ${ }^{22}$. M. Jankovic ${ }^{13} \cdot$ International Berlin-Frankfurt-Münster Study Group Early and Late Toxicity Educational Committee (I-BFM-SG ELTEC)

${ }^{1}$ Epidemiology and Biostatistics Section, Gaslini Children Hospital, Genova,

${ }^{2}$ Department of Psychology, San Diego State University, San Diego, ${ }^{3}$ International Confederation of Childhood Cancer Parent Organization (ICCCPO), c/o VOKK, Nieuwegein, ${ }^{4}$ McMaster University Health Science Centre, Hamilton, ${ }^{5}$ Kinder- und Jugendklinik, Universitätsklinikum Erlangen, ${ }^{6}$ Boyne Research Institute, Drogheda, ${ }^{7}$ Pädiatrische Hämatologie \& Onkologie, Klinik für Kinder-Onkologie, -Hämatologie und Klinische Immunologie, Universitätsklinikum, Heinrich-Heine-Universität Düsseldorf, ${ }^{8}$ Department of Radiation Oncology, University of Pennsylvania, Philadelphia, ${ }^{9}$ Department of Psychology, The University of Sheffield, Sheffield, ${ }^{10}$ Ostschweizerisches Kinderspital, St. Gallen, ${ }^{11}$ UCL Institute of Child Health and Great Ormond Street Hospital for Children NHS Trust, London, ${ }^{12}$ Klinische Abteilung für Pädiatrische Hämatologie/Onkologie , Universitätskinderklinik Graz, ${ }^{13}$ Department of Paediatrics, Hospital San Gerardo, University of Milano-Bicocca, Monza, ${ }^{14}$ Department of Haematology and Oncology, Gaslini Children Hospital, Genova, ${ }^{15}$ Heim Pal Children Hospital, Budapest, ${ }^{16}$ Department of Paediatrics, Academisch Ziekenhuis van de Vrije Universiteit, Brussels, ${ }^{17}$ School of Medicine and Health Sciences, George Washington University, Washington, ${ }^{18}$ Department of Clinical Medicine, Prevention of Biotechnologies, University of Milano-Bicocca, Monza, ${ }^{19}$ Vrije Universiteit Medical Centre, Amsterdam, ${ }^{20}$ Frenchay Hospital, Bristol, ${ }^{21}$ Department of Paediatrics, Rikshospitalet-radiumhospitalet Medical Clinic, Oslo, ${ }^{22}$ Department of Paediatric Oncology, Emma Children's Hospital/Academic Medical Center, Amsterdam

\title{
Langzeitüberlebende einer Krebserkrankung im Kindesalter: Heilung und Betreuung
}

\section{Die Erice-Deklaration}

\section{0-Punkte-Abkommen}

\section{Ziel der Heilung und Nachsorge für ein krebskrankes Kind}

Das langfristige Ziel aller Bemühungen für ein krebskrankes Kind ist seine Heilung, damit es sich zu einer seelisch und körperlich starken Persönlichkeit entwickeln kann, die als Erwachsener voll leistungsfähig und mit einer altersentsprechend optimalen gesundheitsbezogenen Lebensqualität in unserer Gesellschaft integriert ist.

Deutsche Übersetzung des Beitrags: Haupt $\mathrm{R}$, Spinetta JJ, Ban I et al (2007) Long term survivors of childhood cancer: Cure and care. Eur J Cancer 43(12):1778-1780

Veröffentlichung mit freundlicher Genehmigung von Elsevier
1. Der Begriff „Heilung“ bezieht sich auf die ursprüngliche Krebserkrankung (vgl. unten stehende Anmerkung) ohne Berücksichtigung bereits vorhandener Behinderungen oder die Möglichkeit therapiebedingter Nebenwirkungen, die als separate Problematik gewertet werden müssen. Die Überlebenden bedürfen einer individuellen und den Behandlungsrisiken angepassten Langzeitnachsorge. Der Begriff ,geheilt" sollte in der Diskussion über den Gesundheitszustand mit den ehemaligen Patienten benutzt werden, ebenso wie in der brei- ten Öffentlichkeit.[BR]Der Begriff „Langzeitüberlebende“ sollte weiterhin in der wissenschaftlichen Forschung und in der mit ihr zusammenhängenden Literatur eingesetzt werden, um so die Fachleute auf mögliche Spätfolgen und deren Behandlung aufmerksam zu machen.

2. Die Betreuung eines Kindes mit Krebs bedarf einer umfassenden und ehrlichen Aufklärung (abhängig vom Alter und kulturellen Hintergrund) über die Diagnose, die Behandlungsstrategie und die Heilbarkeit der Krankheit.[BR]Die Aufklärung über Heilungschancen eines krebskranken Kindes oder Jugendlichen sollte 
durch den zuständigen pädiatrischen Onkologen, im Einklang mit allen Beteiligten und unter Berücksichtigung individueller Umstände erfolgen.

3. Die Aufklärung der Überlebenden einer Krebserkrankung im Kindesalter und deren Familien hinsichtlich möglicher Risiken ist eine schwierige und sehr anspruchsvolle Aufgabe. Die Informationen sollten in einer leicht verständlichen Sprache und mit einem positiven Hintergrund gegeben werden. Eine effektive Kommunikation verlangt einen hohen Grad zwischenmenschlicher Fertigkeiten. Die Überlebenden und deren Familien haben ein Recht, über ihre Heilungschancen, das Rückfallrisiko sowie die möglichen Spätfolgen der Therapie einschließlich der Entstehung von Zweittumoren mündlich und schriftlich ausführlich informiert zu werden.[BR]Das jeweilige pädiatrisch-onkologische Zentrum (POZ) ist dafür verantwortlich, nach $\mathrm{Ab}$ schluss der onkologischen Therapie eine Zusammenfassung der Krankheitscharakteristika, der durchgeführten Behandlung sowie der aufgetretenen Komplikationen dem Patienten bzw. seinen Angehörigen auszuhändigen. Darüber hinaus sollte dieses Resümee auch Vorschläge bezüglich der Art und des Zeitplans der Nachsorge beinhalten, um die Über- wachung der ursprünglichen onkologischen Erkrankung zu gewährleisten und mögliche Spätfolgen sowohl der Krebserkrankung als auch der onkologischen Therapie frühzeitig zu erfassen.[BR]Nach dem Übergang ins Erwachsenenalter sollte der Langzeitüberlebende von einem Spezialisten betreut werden, der die gesamte Langzeitnachsorge koordiniert. Beim Auftreten von spezifischen Problemen, die als mögliche Therapiespätfolgen betrachtet werden können, sollte der Betroffene an einen mit dem Problem vertrauten Spezialisten überwiesen werden. Dieser hat das Anrecht auf einen vom POZ verfassten Bericht, der die krankheit- und die therapiebedingten Spätfolgen beinhaltet. Darüber hinaus sollte das POZ über Einrichtungen verfügen, die Auskünfte über den Gesundheitszustand aller ehemaligen krebskranken Kinder aufnehmen und archivieren können.

4. Nach der Heilung von ihrer Krebserkrankung im Kindesalter müssen die Patienten im Rahmen einer kontinuierlichen und systematischen Langzeitnachsorge hinsichtlich potenzieller Spätfolgen weiter betreut werden. Um dem nachzukommen, sollte jedes POZ über eine gut strukturierte Spätfolgenklinik verfügen, in der der $\mathrm{Pa}$ tient individuell von einem Spezialistenteam (Pflegepersonal, pädiatri- scher Onkologe, Psychologe, Sozialarbeiter und andere) betreut wird.

5. Um eine adäquate Unterstützung und Beratung der Langzeitüberlebenden einer Krebserkrankung im Kindesalter gewährleisten zu können, sollte das POZ aktuelle Informationen im Rahmen von klinischen Forschungsprojekten sammeln. Die Prioritäten dieser Forschungsagenden sollten vom Nachsorgeteam mit den Überlebenden und ihren Familien festgelegt werden. Eine evidenzbasierte Beratung erfordert das Zusammentragen von Forschungsergebnissen, auf denen mögliche Empfehlungen basiert werden. Die Resultate dieser Forschung sollten den Betreuungsteams, den Überlebenden und deren Familien zur Verfügung gestellt werden, damit diese in die Praxis umgesetzt werden können.

6. Die Überlebenden einer Krebserkrankung im Kindesalter und ihre Familien sollten durch systematische Anstrengungen während und nach der Krebstherapie tatkräftig unterstützt werden. Durch die Versorgung mit altersadäquaten Informationen werden sie ermächtigt, mit den aktuellen und auch zukünftigen Belastungen umgehen zu können.[BR]Die meisten Überlebenden und ihre Familien kommen gut mit ihrer Lebenssituation zurecht. Durch den Ausbau von Anpassungsfähigkeiten und

\title{
Hier steht eine Anzeige.
}

\author{
Springer
}


Information

\section{Teilnehmer am Erice-Symposium}

Epidemiologen

J. Byrne (Leitung, Irland), G. Pastore (Italien), M.G. Valsecchi (Italien), P. Kaatsch (Deutschland),

M.M. Hawkins (Birmingham)

Pflegepersonal

F. Gibson (Leitung, England), M. Bradwell (England), U. Kreichbergs (Schweden), N. Langeveld (Niederlande), A. Thorvildsen (Norwegen), K. Ruccione (USA)

Eltern

M. Chesler (Leitung, USA), I. Ban (Serbien), M. Naafs-Wilstra (Niederlande), M. Serrafero Bonini (Italien), C. Wandzura (Kanada)

Ärzte

G. Masera (Leitung, Italien), R.D. Barr (Kanada), J.D. Beck (Deutschland), J. Bokkerink (Niederlande), G. Calaminus (Deutschland), A. Feldges (Schweiz), G.J. D'Angio (USA), R. Haupt (Italien),

M. Jankovic (Italien), H. Lackner (Österreich), Th. Langer (Deutschland), E. Magyarosy (Ungarn),

R. Maurus (Belgien), L. Massimo (Italien), C. Micalizzi (Italien), A. Moericke (Deutschland),

J. Otten (Belgien), P. Phillipet (Belgien), G. Reaman (USA), C. van den Bos (Niederlande),

A.J.P. Verrman (Niederlande)

Psychologen

J. J. Spinetta (Leitung, USA), C. Eiser (England), C. Favara (Italien), P. Gonzales-Doupe (England),

G. Medin (Spanien), B. Last (Niederlande), P. Massaglia (Italien)

Überlebende

A. Penn (Leitung, England), E. Coenen (Niederlande), An. Cometa (Italien),

M. van de Wettering (Niederlande)

Übersetzung

- A. Feldges LA, em. Ostschweizer Kinderspital, St. Gallen

- J.D. Beck, LESS-Projekt, Kinder- und Jugendklinik, Universitätsklinikum Erlangen

- J. Peeters, LESS-Projekt, Kinder- und Jugendklinik, Universitätsklinikum Erlangen

- H. Lackner, Abteilung für Pädiatrische Hämatologie und Onkologie,

Universitätsklinik für Kinder- und Jugendheilkunde, Medizinische Universität Graz

\section{Korrespondenzadresse}

Prof. Dr. H. Lackner

Abteilung für Pädiatrische Hämatologie/Onkologie

Universitätsklinik für Kinder- und Jugendheilkunde

Medizinische Universität Graz

Auenbruggerplatz 30

A-8036 Graz

herwig.lackner@meduni-graz.at

Stärkung von Bewältigungsstrategien wird eine Widerstandsfähigkeit entwickelt, die den Betroffenen hilft, den zukünftigen Herausforderungen im Leben wirkungs- und vertrauensvoll $\mathrm{zu}$ begegnen und diese $\mathrm{zu}$ überwinden.[BR]Diese Strategien werden besonders bei der Auseinandersetzung mit kritischen Situationen wichtig, wie bei Therapieende, beim Übertritt von der Nachbehandlung zur Langzeitnachsorge und beim Übergang von einer pädiatrischen zu einer auf die Erwachsenenmedizin fokussierten Gesundheitseinrichtung. Durch eine spezielle Kontaktperson lassen sich solche Übergänge erleichtern.
7. Wie bereits erwähnt, ist die Mehrheit der Überlebenden einer Krebserkrankung im Kindesalter relativ gut an ihre Lebenssituation angepasst. Ein Teil von ihnen entwickelt sogar eine ausgeprägte Widerstandsfähigkeit. Im Vergleich zur Allgemeinbevölkerung weisen einige Überlebende jedoch ein erhöhtes Risiko für Probleme auf, die medizinische, psychologische und soziale Hilfe benötigen. Das Gesundheitssystem sollte diesem Umstand Rechnung tragen.

8. Eltern, Geschwister und andere enge Familienmitglieder sollten immer wieder ermuntert werden, sich aktiv an der Diskussion über die Zukunftspläne der Betroffenen sowie die Organisation und Implementierung psychosozialer Interventionen zu beteiligen.[BR]Eltern und Angehörige spielen eine wichtige Rolle beim Informationsaustausch, bei der Unterstützung anderer Betroffener und bei der Gestaltung und Implementierung zukünftiger Dienstleistungen. Eltern und Elternselbsthilfegruppen sollten daher als aktive Mitglieder in das multidisziplinäre Betreuungsteam integriert werden.

9. Die Öffentlichkeit muss wissen und akzeptieren, dass der Krebs im Kindesalter tatsächlich heilbar ist und dass in den letzten 30 Jahren ein signifikanter Fortschritt auf dem Gebiet der onkologischen Therapie stattgefunden hat. So sind heute mehrere hunderttausend Menschen von ihrem Krebs im Kindesalter geheilt. Sie schließen ihre Schul- und Berufsbildung ab und treten als aktive, den Anforderungen des Lebens gewachsene Mitglieder der Gesellschaft ins Erwachsenenalter ein. Eine liberale Gesellschaft sollte sicherstellen, dass Überlebende einen ebenbürtigen $\mathrm{Zu}$ gang zu Erziehung, Anstellung, Versicherung und medizinischer Versorgung haben.

10. Die Ungleichheit der heutigen Behandlungsstrategien und die unterschiedlichen Heilungsraten sowohl innerhalb eines Landes als auch zwischen den Nationen der Staatengemeinschaft basieren vorwiegend auf sozioökonomischen Unterschieden sowie mangelhaften Ressourcen und bleiben eine große Herausforderung für die internationale Gemeinschaft.

\section{Anmerkung}

Es ist nicht möglich, eine exakte Definition für die Heilung festzulegen, die für alle Krebsarten im Kindesalter ihre Gültigkeit behält. Beim Verfassen dieses Dokuments über das Überleben nach einer Krebserkrankung im Kindesalter kam die Gruppe zu folgender Übereinstimmung: Die Heilung bezieht sich nur auf die ursprüngliche Krebserkrankung ohne Berücksichtigung bereits vorhandener Behinderungen oder die Möglichkeit therapiebedingter Nebenwirkungen. Kinder, die wegen einer Krebserkrankung behan- 
delt wurden, können als geheilt betrachtet werden, wenn sie den Zeitpunkt erreicht haben, an dem das Risiko, an ihrer Grundkrankheit zu sterben, nicht mehr größer ist als das allgemeine Sterberisiko von gleichaltrigen Geschwister in der Allgemeinbevölkerung. Für viele maligne Erkrankungen im Kindesalter gilt, dass ein Patient, der eine bestimmte Anzahl von Jahren nach der Diagnosestellung rückfallfrei überlebt hat, als geheilt betrachtet werden kann. Der Zeitpunkt dieser Heilung hängt vom jeweiligen Tumortyp, Tumorstadium und anderen biologischen Faktoren ab. Für einige Tumoren lässt sich diesbezüglich jedoch keine genaue Aussage machen, da bei gewissen Krebsformen genetische Faktoren eine bedeutende Rolle hinsichtlich der Ursache und des Ansprechens der Therapie spielen.

Danksagung. Das Erice-Symposium wurde von der Gaslini Fundation und der San Paolo Fundation, von Buitoni s.p.a. und dem M. Letizia Verga Komitee unterstützt.

Frau C. Manganini wird für ihre ausgezeichnete Sekretariatarbeit gedankt.

Interessenkonflikt. Der korrespondierende Autor gibt an, dass kein Interessenkonflikt besteht.

Monatsschr Kinderheilkd 2009 · 157:346-349 DOI 10.1007/s00112-008-1864-y

\section{R. Haupt · J.J. Spinetta - I. Ban - R.D. Barr - J.D. Beck · J. Byrne - G. Calaminus - E. Coenen · M. Chesler · G.J. D'Angio - C. Eiser · A. Feldges · F. Gibson · H. Lackner · G. Masera - L. Massimo · E. Magyarosy · J. Otten · G. Reaman · M.G. Valsecchi · A.J.P. Veerman · A. Penn · A. Thorvildsen . C. von den Bos - M. Jankovic · International Berlin-Frankfurt-Münster Study Group Early and Late Toxicity Educational Committee (I-BFM-SG ELTEC) Langzeitüberlebende einer Krebserkrankung im Kindesalter: Heilung und Betreuung. Die Erice-Deklaration}

\section{Zusammenfassung}

In den letzten Jahren ist die Anzahl von Patienten, die erfolgreich wegen einer Krebserkrankung im Kindesalter behandelt wurden, stetig angestiegen. Mitglieder des Früh- und Spättoxizitäts- sowie Ausbildungskomitees der internationalen Berlin-Frankfurt-Münster Studien-Gruppe (I-BFM-SG-ELTEC) haben 45 pädiatrische Krebsspezialisten (Onkologen, Psychologen, Pflegepersonal, Epidemiologen, Eltern und Überlebende) aus 13 europäischen Ländern (zusätzlich 5 Experten aus Nordamerika) vom 27.-29.10.2006 nach Erice (Sizilien) eingeladen, um die Lebenssituation der Kinder nach einer Krebserkrankung zu besprechen, eine Definition von Heilung festzu- legen sowie die Notwendigkeit und die Organisation der Nachbeobachtung und Nachsorge zu diskutieren. Es war das erklärte Ziel der Konferenz, mit Hilfe der persönlichen und professionellen Erfahrung der Teilnehmer ein generelles Dokument zu erarbeiten, das die Meinung dieser Arbeitsgruppe zur Heilung und Nachsorge von Überlebenden einer Krebserkrankung im Kindesalter wiedergibt. Dies ist in den 10 Punkten des Erice-Dokuments zusammengefasst.

\section{Schlüsselwörter}

Krebserkrankungen bei Kindern · Heilung . Betreuung · Gesundheit · Lebensqualität

\section{Long-term survivors of childhood cancer: Cure and care. The Erice Statement}

\section{Abstract}

The number of individuals who have successfully completed treatment for a cancer diagnosed during childhood and are entering adulthood has been increasing. Members of the International Berlin-Frankfurt-Munster Early and Late Toxicity Educational Committee (ELTEC) invited 45 paediatric cancer experts - representing oncologists, psychologists, nurses, epidemiologists, parents, and survivors - from 13 European countries, with five additional experts from North America, to Erice, Sicily, on October 27-29, 2006, to discuss the circumstances in which the word "cure" should be used when speaking about children with cancer, and when and why continuing follow-up and care may be required. The objective of the gathering was to generate from the participants' personal and professional experiences an overview statement of the group's philosophy of cure and care of survivors of childhood cancer. The 10 points reflect what the group considers essential for the survivors' cure and care.

\section{Keywords}

Childhood cancer - Childhood cure .

Childhood care · Health · Quality of life 